\title{
Ambiente letrado y estrategias didácticas en la educación preescolar chilena
}

Literate Environment and Didactic Strategies in Chilean Preschool Education Environnement lettré et stratégies didactiques dans l'éducation préscolaire chilienne

Ambiente letrado e estratégias didáticas na educação infantil chilena

Fecha de recepción: 20 DE MAYO DE 2013/ fecha de aceptación: 5 DE OCTUBRE DE 2013

Encuentre este artículo en http://magisinvestigacioneducacion.javeriana.edu.co/

doi:10.11144/Javeriana.M6-13.ALED

Escrito por Pelusa Orellana-García UNIVERSIDAD DE LOS ANDES SAntiago, Chile porellan@uandes.cl

Carolina Melo-Hurtado UNIVERSIDAD DE VIRGINIA Santiago, Chile carolina@virginia.edu

\section{Resumen}

El presente estudio analiza la calidad del ambiente letrado y de las estrategias didácticas usadas por educadoras de párvulos en 147 salas de preescolar de distintos niveles socioeconómicos en Chile. Para recopilar los datos se usó la herramienta ELLCO (Early Language and Literacy Observation Tool), que incluye autoinformes, listas de cotejo, entrevistas y escalas de valoración. Los resultados muestran que la calidad del ambiente letrado y de las estrategias didácticas empleadas es baja y que existen diferencias significativas entre establecimientos de distinto nivel socioeconómico.

\section{Transferencia a la práctica}

Los hallazgos reafirman la importancia de contar con un adecuado ambiente letrado y de utilizar estrategias didácticas efectivas en el desarrollo de los procesos de alfabetización en la educación inicial. Esta importancia, fundamentada desde la teoría, se constata al observar ciertas características y estrategias cuya intención es promover el lenguaje oral, la lectura y la escritura, en un contexto donde el niño puede interactuar con materiales que propician una preparación acorde con el desarrollo de habilidades que contribuirán a su Palabras clave descriptor Alfabetización (educación), lectura, escritura, métodos de enseñanza.

Para citar este artículo / To cite this article / Pour citer cet article / Para citar este artigo /

Orellana-García, P., \& Melo-Hurtado, C. (2014). Ambiente letrado y estrategias didácticas en la educación preescolar chilena. magis, Revista Internacional de Investigación en Educación, 6 (13), 113-128. 
Key words author

Early Literacy, Reading and Writing,

Literate Environment, Didactic Strategies.

\section{Key words plus}

Alphabetization (Education), Reading, Writing, Educational Method

\section{Abstract}

This study analyzes the quality of literate environment and didactic strategies used by preschool teachers in 147 establishments of different socioeconomic environments in Chile. For data collection, the ELLCO (Early Language and Literacy Observation Tool) was used, which includes self-reports, checklists, surveys and rating scales. The results show that the quality of literate environment is low and that there are significant differences between establishments of different socioeconomic level.
Transference to practice

The findings confirm the importance of adequate literate environments and effective didactic strategies for the development of literacy processes of preschool education. This importance, described in theoretical work, is corroborated when observing certain characteristics and strategies that aim to stimulate oral language, reading and writing, in a context where the child interacts with materials that help develop skills in line with learning processes at school.

\section{Mots clés auteur}

Alphabétisation précoce, lecture et écriture, environnement lettré, stratégies didactiques.

Mots clés descripteur

Alphabétisation, lectura, écriture, étude et enseignement.

\section{Résumé}

Cette étude analyse la qualité de l'environnement lettré et des stratégies didactiques utilisés par les éducatrices de préscolaire en 147 salles de préscolaire de différents niveaux socioéconomiques au Chili. Pour réunir les données on a utilisé l'outil ELLCO (Early Language and Literacy Observation Tool), qui inclut auto-rapports, listes de confrontation, entretiens et échelles d'évaluation. Les résultats montrent que la qualité de l'environnement lettré et des stratégies didactiques employées est baisse et qu'existent différences significatives entre les établissements de différent niveau socioéconomique.

\section{Transfert à la pratique}

Les trouvailles réaffirment l'importance de compter avec un adéquat environnement lettré et d'utiliser les stratégies didactiques effectives dans le développement des processus d'alphabétisation dans l'éducation initiale. Cette importance est fondée depuis la théorie, puisque on constate en observant certaines caractéristiques et stratégies dont l'intention est promouvoir le langage oral, la lecture et l'écriture, dans un contexte où l'enfant peut interagir avec les matériaux qui favorisent une préparation en accord avec le développement d'habilités qui contribueront avec son apprentissage scolaire.

\section{Palavras-chave autor}

Alfabetização precoce, leitura e escrita, ambiente letrado, estratégias didáticas.

Palavras-chave descritor Alfabetização, leitura, escrita, estudo ensino.

\section{Resumo}

O presente estudo analisa a qualidade do ambiente letrado e das estratégias didáticas usadas por educadoras em 147 salas de educação infantil de diferentes níveis socioeconômicos no Chile. Para reunir os dados se usou a ferramenta ELLCO (Early Language and Literacy Observation Tool), que inclui autorrelatórios, listas de cotejo, entrevistas e escalas de avaliação. Os resultados mostram que a qualidade do ambiente letrado e das estratégias didáticas empregadas é baixa e que existem diferenças significativas entre estabelecimentos de diferentes níveis socioeconômicos.

\section{Transferência à prática}

Os resultados confirmam a importância de contar com um adequado ambiente letrado e de utilizar estratégias didáticas efetivas no desenvolvimento dos processos de alfabetização na educação infantil. Esta importância, fundamentada a partir da teoria, se constata ao observar certas características e estratégias cuja intenção é promover a linguagem oral, a leitura e a escrita, num contexto onde a criança pode interagir com materiais que propiciam uma preparação conforme o desenvolvimento de habilidades que contribuirão com a sua aprendizagem escolar. 
La educación preescolar busca, entre otras cosas, sentar una base sólida de hábitos y disposiciones que permitan a los niños una apropiada inserción en el sistema escolar. Uno de los aspectos clave de dicha base es la adquisición de habilidades y conocimientos necesarios para la alfabetización posterior $y$, en este contexto, la existencia de un ambiente letrado rico en estímulos del lenguaje resulta fundamental. Numerosos estudios han comprobado que los niños que durante la educación preescolar han estado expuestos a una amplia variedad de estímulos impresos y que han comenzado a experimentar con la lectura y la escritura en entornos significativos están mejor preparados para enfrentar la enseñanza formal de la lectura y la escritura (Snow, Burns \& Griffin, 1998; Melhuish, Sylva, Sammons, Siraj-Blatchford, Taggard \& Phan, 2008). De hecho, muchas habilidades de alfabetización emergente, como la conciencia fonológica, el conocimiento del nombre de las letras del alfabeto y la conciencia de lo impreso son predictores importantes del posterior dominio lector (Greenfield Spira, Storch Brachen \& Fischel, 2005; Castejón, González-Pumariega \& Cuetos, 2011; Storch \& Whitehurst, 2002, entre otros). Estos hallazgos refuerzan la relevancia de una interacción temprana con la lectura y la escritura, en especial para aquellos niños que provienen de contextos en donde no suelen estar expuestos a este tipo de estímulos.

Del mismo modo, las estrategias didácticas utilizadas para estimular la alfabetización inicial pueden promover un adecuado desarrollo de la lectura y la escritura iniciales. Dentro de estos aspectos se incluye la calidad de las interacciones que facilitan la adquisición del lenguaje oral, precursor de la lectura y la escritura (Guo \& Justice, 2010; Roskos \& Neuman, 2001; Domitrovich, Gest, Gil, Bierman, Welsh \& Jones, 2008) y la incorporación de ciertos conceptos básicos de alfabetización por medio de la lectura y la escritura con el adulto, quien suele modelar comportamientos relacionados con leer y escribir en ambientes reales (Snow et al., 1998; Wasik \& Bond, 2001).

La presente investigación tiene por objetivo describir y analizar la calidad del ambiente letrado y las estrategias usadas en 147 salas de educación preescolar de distintos niveles socioeconómicos en una extensa región urbana de Chile. Un segundo objetivo es determinar si existe un efecto diferenciador en la calidad del ambiente y las estrategias didácticas según el tipo de establecimiento. Para abordar estas interrogantes se usó una versión en español de ELLCO (Early Language and Literacy Classroom Observation) (Smith, Brady \& Clark-Chiarelli, 2002), que permitió recopilar datos mediante observación directa y analizarlos en forma cuantitativa. El empleo de instrumentos como ELLCO para la observación de clases en educación preescolar y básica es relativamente conocido (Lo Casale-Crouch, Konold, Pianta, Howes, Burchinal \& Bryant, 2007; Senechal, Oullette \& Rodney, 2006; Pianta, LaParo \& Hamre, 2008). Sin embargo, en América Latina la mayoría de las investigaciones acerca de estrategias didácticas de observación se ha focalizado en aspectos como apoyo emocional, organización del aula y apoyo pedagógico (Treviño, Toledo \& Gempp, 2013), pero no se han encontrado estudios que aborden la calidad del ambiente letrado y las estrategias didácticas utilizadas por las educadoras para promover el aprendizaje de la lectoescritura.

ELLCO permite obtener una medida global de la calidad de las salas de educación preescolar, la cual ha demostrado tener efectos positivos en la adquisición de habilidades de lenguaje y alfabetización (Cunningham, Zibulsky \& Callahan, 2009). Esta calidad global se define como aquella que mejor apoya el desarrollo positivo de los niños en función de actividades de aprendizaje adecuadas a edad, ambientes seguros y saludables e
Descripción del artículo | Article description | Description de l'article | Artigo descrição

Este artículo de investigación analiza los resultados de la evaluación de la calidad del ambiente letrado y de las estrategias didácticas empleadas por educadores de párvulos en Chile. Se constata que persisten problemas de calidad en ambos aspectos. Las razones que explican esta baja calidad se analizan tanto desde la perspectiva de la formación docente como desde la falta de conocimiento acerca de lo que es un buen ambiente letrado. 
interacciones positivas y adecuadas entre los adultos y los niños (Smith et al., 2002). La calidad del ambiente letrado incluye el entorno y las relaciones sociales en las que los niños intervienen al estar en una sala (Smith \& Dickinson, 2002). En general, son pocos los estudios que han examinado el entorno físico de la sala de clases así como las estrategias didácticas, con el fin de determinar si contribuyen al desarrollo de la alfabetización.

\section{Ambiente letrado y estrategias didácticas en educación preescolar}

La calidad de las experiencias de alfabetización temprana se relaciona de manera significativa con el posterior desempeño académico de los estudiantes (Connor, Morrison \& Slominski, 2006). Estas experiencias se manifiestan de diversas formas, como las interacciones entre los estudiantes y los educadores, el entorno rico en texto impreso y las formas de iniciar a los niños en la lectura y la escritura (McGinty, Justice, Piasta, Kaderavek \& Fan, 2012). Entre los aspectos que afectan positivamente la calidad de las experiencias de alfabetización temprana se destaca el lenguaje oral -manifestado en la presencia de un diálogo o intercambio verbal-que plantee desafíos tanto cognitivos como lingüísticos, además de la lectura dialogada y el trabajo del vocabulario (Villalón, Rojas, Föster, Valencia, Cox \& Volante, 2011). Cuando las conversaciones que los educadores generan en la sala de clase surgen de preguntas abiertas que estimulan el diálogo y las ideas, se amplía el espectro verbal y cognitivo de los niños (Gjems, 2013; Pressley, Rankin \& Yokoi, 1996). Ayudarles a expresarse con palabras nuevas facilita la adquisición de conceptos y enriquece el léxico y la estructura sintáctica de los niños. Por el contrario, un entorno lingüístico que no propicie una conversación más elaborada impide que adquieran mayor precisión léxica, asocien ideas y conceptos con palabras específicas o establezcan conexiones entre conocimientos previos y aquello que van internalizando a medida que dialogan.

Numerosos estudios confirman la conveniencia de introducir tempranamente algunas habilidades como identificar letras y palabras, a fin de preparar mejor a los niños para el aprendizaje de la lectura (Rupley, Blair \& Nichols, 2009). Los modelos que integran aspectos como la conciencia fonológica y el reconocimiento de letras mediante la lectura y la discusión de libros pueden optimizar la alfabetización en los niños y ayudarles a decodificar y comprender (Justice \& Kaderavek, 2004; Skibbe, Connor, Morrison \& Jewkes, 2011; Justice, Kaderavek, Fan, Sofka \& Hunt, 2009).

Las experiencias de alfabetización temprana adquieren mayor relevancia para aquellos niños que carecen de una estimulación verbal y cognitiva necesaria en el hogar, para insertarse luego en el mundo letrado. Varias investigaciones han constatado que una educación preescolar de calidad puede compensar la falta de exposición al lenguaje, el vocabulario reducido o una expresión oral poco estimulada (Whitehurst \& Lonigan, 1998; Zill \& Resnick, 2006; Mol \& Bus, 2011; Justice, Meier \& Walpole, 2005). En este sentido, poder contar con una educación preescolar cuyo objetivo principal sea desarrollar el lenguaje y la alfabetización ha de ser tarea prioritaria de cualquier sociedad que busque mejorar las condiciones de aprendizaje de sus estudiantes, sobre todo de aquellos que parten con notorias desventajas lingüísticas y cognitivas (Heckman, 2004).

Alcanzar estos propósitos de alfabetización depende en gran medida del grado de conocimiento y uso que los educadores tengan acerca de los procesos que intervienen en el desarrollo de las habilidades del lenguaje, de la lectura y la escritura (Neuman \& Cunningham, 2009). Se ha 
observado que los educadores de preescolar no siempre cuentan con un conocimiento acabado y actualizado de la importancia que estos factores cobran en la promoción de la alfabetización temprana y sus consecuencias (Cunningham et al., 2009; Mather, Bos \& Babur, 2001; Bravo, Villalón \& Orellana, 2004).

\section{La educación inicial en Chile y el desarrollo de la alfabetización inicial}

Chile es un país con altos índices de alfabetización, con apenas un $3,2 \%$ de población que no sabe leer ni escribir. Lo anterior es resultado de importantes campañas de educación de adultos y de una ampliación de la cobertura de la educación escolar básica en las últimas cuatro décadas. Durante el gobierno de la presidenta Bachelet se estableció como meta aumentar significativamente la cobertura de la educación preescolar, lo cual benefició a un gran porcentaje de la población. La inversión en educación, tanto en infraestructura como en perfeccionamiento docente, continúa incrementándose en todos los niveles educacionales. No obstante, en los últimos veinte años han persistido los bajos desempeños de los estudiantes chilenos. Las evaluaciones PISA muestran que dos tercios de los estudiantes de 15 años no logra comprender textos básicos y que casi un $44 \%$ de estos se concentra en la educación municipalizada o pública (Mineduc, 2011).

Cabe destacar que la brecha en resultados de comprensión lectora según nivel socioeconómico es enorme: si en los colegios públicos la mitad de los alumnos no comprende lo que lee, en los privados el porcentaje es de apenas $8 \%$ (Mineduc, 2011) y, lo que es más grave, esta brecha se ensancha a medida que los alumnos avanzan en sus estudios. Por otra parte, se ha observado una alta correlación entre el desempeño lector en cuarto año básico y la calidad de la enseñanza y los materiales usados por los profesores de educación preescolar (Lira \& Rodríguez, 1997; Centro de Estudios de Desarrollo y Estimulación Psicosocial, 1997; Villalón, Suzuki, Herrera \& Mathiesen, 2002). Otros estudios que profundizan en el tema muestran que en estas salas de clases no existe material literario de calidad (Strasser, Lissi \& Silva, 2009; Villalón, Silva, Razmilic \& Swartz, 2005, Eyzaguirre \& Fontaine, 2008; Eyzaguirre \& Le Foulon, 2002), lo que dificulta el logro de estos objetivos. A modo de ejemplo, Strasser y otros autores (2009) observaron que más de la mitad del tiempo que los niños pasan en las salas de preescolar públicas y privadas de la educación chilena se destina a actividades no relacionadas con la enseñanza.

La escasez de ambientes letrados que estimulen el desarrollo del lenguaje no es el único obstáculo que enfrenta la educación preescolar del país. La calidad de la formación inicial en educación de párvulos muestra falencias considerables respecto a contenidos fundamentales para comprender el alcance y la importancia de la alfabetización temprana (Villalón et al., 2011; García-Huidobro, 2006; Strasser \& Lissi, 2009). Se aprecia una falta de actualización de conocimientos de psicología del desarrollo, del aprendizaje de lenguaje y de la neurociencia. A lo anterior se debe agregar la necesidad de contar con mayor producción científica nacional que evidencie la relevancia del tema de la calidad sobre el de la cobertura y las consecuencias que la carencia de alfabetización temprana y un ambiente letrado de calidad tendrán para los miles de escolares chilenos que año tras año ingresan a la educación básica sin las herramientas necesarias para avanzar a mayor ritmo en el aprendizaje de la lectura y la escritura. 


\section{Metodología}

En el presente estudio se intenta conocer la calidad de la alfabetización preescolar en Chile en función de dos variables: la calidad del ambiente letrado y las estrategias didácticas para el desarrollo de habilidades de alfabetización inicial. Junto con ello, analizamos las relaciones entre ambas variables, para identificar posibles diferencias en la calidad de las mismas según los tipos de establecimientos: particulares pagados, municipalizados y particulares subvencionados, que atienden a estudiantes provenientes de hogares de nivel socioeconómico alto, medio y bajo, respectivamente.

Para abordar estas preguntas se visitaron 147 salas preescolares (jardines infantiles y preescolares de colegios) en un sector urbano chileno. Con una versión traducida de Early Childhood Language and Litearcy Observation Toolkit (Smith et al., 2002) se evaluó la calidad del ambiente letrado y de las estrategias didácticas utilizadas. Se examinaron aspectos funcionales, de interacción, de lenguaje y de alfabetización relacionados con dicho ambiente, lo que entregó información cualitativa y cuantitativa. Además, se examinó la correlación entre ambas variables y, para detallar las diferencias entre establecimientos de distinta dependencia, se hicieron análisis de varianza.

Las categorías de análisis fueron: tipo de establecimiento, calidad del ambiente letrado y calidad de las estrategias didácticas. Las categorías de observación para el tipo de establecimiento fueron tres y se explican en la sección "Participantes". Para calidad del ambiente letrado se utilizaron los aspectos en la pauta de ambiente letrado, descritos en la sección "Instrumentos". En dicha sección también se presentan los aspectos relativos a la calidad de estrategias didácticas según la Escala de calificación de actividades de clase y observación de clases, con sus secciones Lenguaje, Alfabetización y currículo y Ambiente general de la sala de clases.

\section{Participantes}

En total, fueron visitadas y evaluadas 147 salas de clase pertenecientes a 72 establecimientos educacionales de un amplio sector urbano chileno, en función de la calidad de su ambiente letrado y de las estrategias didácticas usadas en el momento de la visita. De las salas observadas, 67 eran de Prekínder (4 a 5 años), 87 correspondían a Kínder (5 a 6 años), dos integraban Prekínder con Kínder y solo una correspondía a Transición (3 a 4 años). Se procuró obtener una muestra que reflejase proporcionalmente la distribución de la población escolar chilena por tipo de dependencia, ya que esta distribución corresponde a tres niveles socioeconómicos. Así, por ejemplo, los niños provenientes de hogares de nivel socioeconómico bajo asisten, en su mayoría, a establecimientos municipalizados, mientras los de nivel socioeconómico medio a establecimientos particulares subvencionados y los de nivel socioeconómico alto a establecimientos pagados. Cabe mencionar que los establecimientos municipalizados son financiados por el Estado y los subvencionados reciben financiamiento parcial mediante la llamada subvención escolar. Los establecimientos particulares pagados son cubiertos íntegramente por los padres.

En la actualidad, un $43 \%$ del total de la población escolar chilena asiste a establecimientos municipales y en las instituciones particulares subvencionadas se educa un $47 \%$ de la población escolar. Por último, cerca de un $10 \%$ de los estudiantes chilenos asiste a establecimientos particulares pagados. 
La Tabla 1 describe la distribución de la muestra en los distintos tipos de establecimientos.

Tabla 1.

Características sociodemográficas

\begin{tabular}{lcccc}
\hline \multicolumn{1}{c}{ Dependencia } & Municipal & Subven. & Particular & Total \\
\hline Total salas observadas & 34 & 74 & 39 & 147 \\
\hline Porcentaje del total observado & $24 \%$ & $50 \%$ & $26 \%$ & $100 \%$ \\
\hline Número de establecimientos & 10 & 27 & 35 & 72 \\
\hline NSE & bajo & medio & alto & \\
\hline Promedio de alumnos por sala & 25 & 32 & 24 & 27 \\
\hline Promedio de adultos por sala & 2 & 2 & 2 & 2 \\
\hline \hline
\end{tabular}

Fuente: elaboración propia

\section{Instrumentos}

Se utilizó una traducción de Early Language and Literacy Classroom Observation Toolkit (ELLCO). Este instrumento consta de tres secciones que permiten medir aspectos relacionados con el lenguaje, la alfabetización y su desarrollo. La primera parte se denomina Pauta de ambiente letrado y en ella se evalúa la presencia de material adecuado para trabajar lectura y escritura. Consta de doce ítems asociados con la presencia y el uso de libros y doce ítems relacionados con el uso y la presencia de material de escritura. Para facilitar la observación de dichas características, el instrumento considera los siguientes indicadores: 1) existencia de un área específica de lectura en la sala de clases; 2) selección y uso de libros; 3) existencia de material de escritura, y 4) evidencia de escritura infantil en la sala. A modo de ejemplo, se pregunta si la sala cuenta con un lugar dedicado con exclusividad a la lectura de libros, si están clasificados según su dificultad o si la sala cuenta con un alfabeto visible para los alumnos. Los veinticuatro ítems de esta sección tienen un puntaje máximo de 41 puntos.

La segunda parte se denomina Escala de observación de clase y comprende catorce preguntas en las que el observador debe calificar, a partir de una escala de 5 puntos, una serie de aspectos referentes a la sala de clases, en donde 1 corresponde a deficiente y 5 a ejemplar. Las observaciones de clase, evaluadas según la subescala, se examinan en función de dos componentes: Lenguaje, alfabetización y currículo, con un máximo de 30 puntos y el Ambiente general de la sala de clases, con un máximo de 40 puntos. Dentro de los aspectos a revisar están la organización, las estrategias de manejo y el currículo de lenguaje, entre otros. A continuación de la Escala de observación de clase hay un breve cuestionario de respuesta abierta que permite complementar lo observado. Este instrumento recoge información cualitativa y no tiene puntaje asignado. La tercera parte de ELLCO es una Escala de calificación de las actividades de alfabetización, que consta de nueve ítems que evalúan la presencia y la calidad de las actividades relacionadas con lenguaje, lectura y escritura. Por ejemplo, se cuantifica el número de minutos durante los cuales la educadora leyó a sus alumnos o si se observó a niños que intentaban escribir letras o palabras. La escala tiene un máximo de 12 puntos, 8 de los cuales corresponden a actividades de lectura y 4 a escritura. 
Validez. y confiabilidad del instrumento

En su versión original ELLCO está validado en contextos de habla inglesa. La validez del proceso de traducción al español se realizó para establecer la equivalencia semántica que asegure que el significado de cada ítem es el mismo en cada cultura después de la traducción. Para ser usado en el presente estudio, el texto original fue traducido mediante el método de back-translation (Brislin, 1970) y un panel de jueces expertos.

Una vez establecida la validez del proceso de traducción a español, se hizo un pilotaje en un establecimiento de educación prebásica para calibrar a los observadores. Se revisaron salas en grupos de cuatro evaluadores y un supervisor. Luego de examinar una misma sala de clases durante una mañana, esas cinco personas compararon sus resultados, para verificar que no hubiese diferencias en la interpretación de los ítems de ELLCO y para identificar cualquier discrepancia o confusión debido a un error de traducción. Se compararon los puntajes por ítem y se logró establecer un índice kappa de Cohen de 0,8 entre los observadores, lo cual demuestra un buen índice de confiabilidad.

Los análisis de confiabilidad entre los ítems de cada sección permitieron determinar la consistencia interna entre las distintas subescalas de ELLCO. Para la Pauta de ambiente letrado se obtuvo un alpha de Cronbach de 0,78; para la Escala de observación de clases, de 0,76 y para la Escala de calificación de actividades de alfabetización se obtuvo un alpha de 0,83 . Al establecer una consistencia interna, es posible confirmar que los ítems de la versión en español corresponden al mismo constructo teórico que sus equivalentes en inglés, ya que los índices del alfa de Cronbach están entre 0,76 y 0,83, lo que constituye un grado aceptable.

Para la recolección de datos se reclutaron a 36 observadores, quienes fueron capacitados por el equipo investigador para utilizar ELLCO. Los observadores evaluaron la calidad del ambiente letrado en distintas salas de clase durante un día completo. Cada observador examinó cinco salas de clase, pero se obtuvieron 147 observaciones debidamente completadas.

\section{Resultados}

Los resultados del estudio se presentan a partir de tres tipos de análisis. En primer lugar, se efectúa un examen preliminar para caracterizar los resultados de frecuencia, media y desviación estándar. Luego, para determinar la calidad del ambiente letrado y las estrategias didácticas, se revisaron las correlaciones. Por último, para verificar la existencia de diferencias significativas entre los establecimientos de distinta dependencia, se hicieron análisis de varianza.

\section{Ambiente letrado}

Los puntajes registrados en la Pauta de ambiente letrado muestran que en las salas visitadas había una cantidad insuficiente de materiales de buena calidad, según los criterios definidos en el instrumento. A modo de ejemplo, la media para el apartado "Libros" (que incluye área para los libros, selección y uso de los mismos) fue de 5,13 (rango 0-12; SD 3,286), de un total de 20 posibles puntos. En el caso de los "Materiales de escritura", el promedio alcanzó 8,83 (rango 1-17; SD 3,449) de un máximo de 21. El puntaje promedio total de la Pauta fue de 13,96 (rango 3-29; SD $5,719)$ de un máximo de 41 puntos. En la Tabla 2 se presentan las estadísticas descriptivas para esta sección del instrumento.

Tabla 2.

Estadísticas descriptivas para la pauta de ambiente letrado

\begin{tabular}{lccc}
\hline & Rango & Media & SD \\
\hline Subtotal libros & $0-12$ & 5,33 & 3,286 \\
\hline $\begin{array}{l}\text { Subtotal materiales } \\
\text { de escritura }\end{array}$ & $1-17$ & 8,83 & 3,449 \\
\hline Total Pauta & $3-29$ & 13,96 & 5,710 \\
\hline \hline
\end{tabular}

Fuente: elaboración propia

De acuerdo con los parámetros establecidos en ELLCO, en el caso de la variedad y calidad se observó una media de 0,09 (rango 1 a 3) para el ítem de ELLCO que pregunta si hay libros con distinto grado de dificultad; de 0,28 (rango 1 a 3) para el ítem que pregunta si existían libros de temas diversos y de 1,16 para el ítem referente a la cantidad de libros de tipo informativo.

Respecto al material para escritura, en la mayoría de las observaciones se encontró solo lápiz y papel y en un $77 \%$ de las salas no existía un lugar acondicionado como centro o área de escritura. Se entiende como tal a un lugar que cuente con una mesa y silla, materiales de escritura o un computador en el que los alumnos puedan escribir. En apenas un tercio de las salas visitadas había evidencia de escritura realizada por los niños publicada en ficheros o en los muros de la sala. Se entiende por trabajos escritos cualquier atisbo de escritura emergente, así como también registros hechos por la educadora a partir de discusiones grupales, recuentos de lectura $u$ otras formas de dictado (Smith et al., 2002). En la Tabla 3 se presentan las medias para material y escritura en sala según tipo de establecimiento, entre los cuales no se observaron diferencias significativas. 
Tabla 3.

Diferencias en calidad de material de escritura según tipo de establecimiento

\begin{tabular}{lcc}
\hline \multicolumn{1}{c}{ Tipo } & $\begin{array}{c}\text { Subtotal materiales } \\
\text { de escritura } \\
\text { M (SD) }\end{array}$ & $\begin{array}{c}\text { Subtotal } \\
\text { escritura en sala } \\
\text { M (SD) }\end{array}$ \\
\hline Particular & $4,82(1,502)$ & $4,15(2,289)$ \\
\hline Subvencionado & $5,07(1,484)$ & $4,23(2,508)$ \\
\hline Municipalizado & $4,35(2,403)$ & $3,29(1,784)$ \\
\hline \hline
\end{tabular}

Fuente: elaboración propia

\section{Estrategias didácticas}

Para determinar la calidad de las estrategias didácticas se usó la Escala de calificación de las actividades de alfabetización del instrumento, así como también los puntajes de las observaciones de clase. En la Tabla 4 se presentan las descriptivas para las variables observadas, que están muy por debajo del puntaje total. En promedio, en la Escala de actividades de alfabetización las salas observadas obtienen un puntaje de 4,98 de un máximo de 12. En la sección Escritura, un promedio de 1,75 de un total de 4. Las salas observadas alcanzan en promedio 40,52 puntos distribuidos así: 23,39 puntos en Lenguaje, alfabetización y currículo y 17,14 para el Ambiente general de la sala de clases. Estos resultados se complementan con datos como que en $40 \%$ de las salas observadas, las educadoras leyeron cuentos a los niños por menos de cinco minutos, aunque se apreciaron algunas diferencias según tipo de establecimiento, por ejemplo, las desigualdades resultaron ser estadísticamente significativas para el número total de minutos dedicados a leer cuentos a los niños $F(2,144)=3,373 ; p=037$, pero no para la cantidad de sesiones de lectura grupal ni para la cantidad de libros leídos a los niños durante las observaciones.

Mediante la observación de clases y su posterior análisis fue posible recopilar información acerca de las actividades que las educadoras realizaban con los niños en relación con la alfabetización inicial. En la Escala de evaluación de las actividades de alfabetización se obtuvo una media de 4,98 puntos de un total de 12, lo que se desagrega en 3,23 de un máximo de 8 para las actividades relacionadas con lectura y de 1,75 de un máximo de 4 para las actividades relacionadas con escritura.

Tabla 4.

Estadísticas descriptivas para la observación de clases y la escala de actividades de alfabetización

\begin{tabular}{lccc}
\hline & Rango & Media & DS \\
\hline Subtotal Ambiente general de la sala de clase & $6-24$ & 17,14 & 4,064 \\
Subtotal Lenguaje, alfabetización y currículo & $10-37$ & 23,39 & 6,056 \\
Total Observación de clase & $17-61$ & 40,52 & 9,409 \\
\hline Subtotal Lectura de libros & $0-8$ & 3,23 & 2,239 \\
Subtotal Escritura & $0-4$ & 1,75 & 1,494 \\
Total Escala de actividad de alfabetización & $0-12$ & 4,98 & 2,932 \\
\hline
\end{tabular}

Fuente: elaboración propia 


\section{Relación entre la calidad del ambiente de enseñanza y las prácticas utilizadas por las educadoras chilenas}

En la Tabla 5 se presentan los coeficientes de correlación obtenidos a partir de las variables de calidad del ambiente y de las estrategias didácticas observadas y registradas en la Escala de evaluación de las actividades de alfabetización. No se observaron correlaciones estadísticamente significativas entre ambas variables, lo que sugiere que, en general, el ambiente y sus materiales (presencia de un rincón de libros, variedad en la selección de libros, materiales de escritura y presencia de material escrito por los alumnos en la sala) no garantizan la calidad de las estrategias usadas por las educadoras ni la carencia de ella. Sí se observó una correlación positiva de 0,346 $(p<0,000)$ entre los puntajes totales de la observación de salas de clase y la Escala de evaluación de actividades de alfabetización.

Tabla 5.

Consistencia interna. Correlaciones de Pearson entre las distintas subescalas y sus componentes

\begin{tabular}{|c|c|c|c|c|c|c|c|c|c|}
\hline & 1 & 2 & 3 & 4 & 5 & 6 & 7 & 8 & 9 \\
\hline 1. Subtotal Libros & -- & $0,437^{* *}$ & $0,840^{* *}$ & 0,025 & 0,050 & 0,043 & 0,144 & 0,103 & $0,162^{*}$ \\
\hline 2. Subtotal Materiales escritura & & -- & $0,856^{* *}$ & $-0,061$ & $-0,107$ & $-0,095$ & $-0,093$ & 0,051 & $-0,045$ \\
\hline 3. Pauta de Ambiente letrado & & & -- & $-0,022$ & $-0,036$ & $-0,033$ & 0,026 & 0,090 & 0,066 \\
\hline $\begin{array}{l}\text { 4. Total Ambiente general } \\
\text { de la sala de clase }\end{array}$ & & & & -- & $0,718^{* *}$ & $0,894^{* *}$ & $0,282^{* *}$ & 0,160 & $0,297^{\star *}$ \\
\hline $\begin{array}{l}\text { 5. Subtotal Lenguaje, } \\
\text { alfabetización y currículo }\end{array}$ & & & & & -- & $0,954^{* *}$ & $0,323^{* *}$ & $0,181^{*}$ & $0,339^{* *}$ \\
\hline 6. Total Observación de clase & & & & & & -- & $0,329^{* *}$ & $0,186^{*}$ & $0,346^{* *}$ \\
\hline 7. Subtotal Lectura libros & & & & & & & -- & $0,202^{*}$ & $0,867^{* *}$ \\
\hline 8. Subtotal Escritura & & & & & & & & -- & $0,664^{* *}$ \\
\hline $\begin{array}{l}\text { 9. Total Escala de actividades } \\
\text { de alfabetización }\end{array}$ & & & & & & & & & -- \\
\hline
\end{tabular}

Fuente: elaboración propia

* Correlación significativa a 0,05 (2 colas). ** Correlación significativa a 0,01 (2 colas).

\section{Diferencias de calidad en el ambiente y las estrategias didácticas usadas por educadoras chilenas según nivel socioeconómico}

Los resultados de la Anova para más de dos grupos mostraron diferencias en la calidad tanto del ambiente como de las estrategias de enseñanza en los tres tipos de establecimientos que imparten educación preescolar en Chile. Para ello se compararon las medias de cada sección de ELLCO en los tres tipos de establecimientos. En la Figura 1 es posible apreciar de manera comparativa la dispersión de los puntajes de las variables en los distintos establecimientos. 
Figura 1.

Pauta de ambiente letrado y escala de actividades de alfabetización según tipo de establecimiento

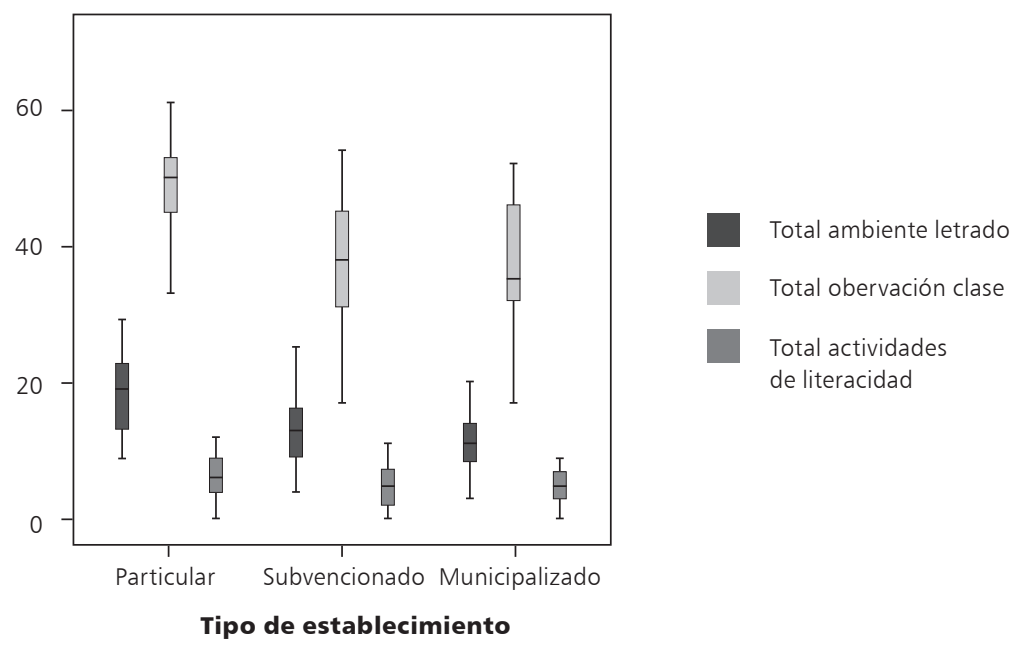

Fuente: elaboración propia

La Tabla 6 muestra los valores medios para cada una de las variables observadas según tipo de establecimiento. Según la Pauta de ambiente letrado, la calidad de este difiere significativamente entre los tres tipos de establecimiento, $F(2,144)=20,918, p=0,000$. Se realizaron además comparaciones post hoc mediante el test de Tukey, lo que permitió establecer que los colegios privados $(\mathrm{M}=18,38 ; 95 \mathrm{Cl}[3,28 ; 8,03])$ tienen ambientes letrados significativamente superiores a los de establecimientos de tipo subvencionado $(\mathrm{M}=11,56 ; 95 \mathrm{Cl}[11,58 ; 13,88])$ y de municipalizados $(\mathrm{M}=$ $11,56 ; 95 \mathrm{Cl}[9,98 ; 13,13])$. Sin embargo, no se observaron diferencias estadísticamente significativas entre subvencionados y municipalizados.

Tabla 6.

Medias para pauta de ambiente letrado según nivel socioeconómico

\begin{tabular}{llcc} 
& & Media & DS \\
\cline { 3 - 4 } Pauta de ambiente letrado. Total & Est. particulares & 18,38 & 5,664 \\
& Est. subvencionados & 12,73 & 4,961 \\
& Est. municipalizados & 11,56 & 4,514 \\
& Total & $\mathbf{1 3 , 9 6}$ & $\mathbf{5 , 7 1 0}$ \\
\hline Observación de clases. Total & Est. particulares & 41,10 & 8,599 \\
& Est. subvencionados & 40,88 & 9,539 \\
& Est. municipalizados & 39,09 & 10,122 \\
\hline Escala de actividades de alfabetización. Total & Est. particulares & $\mathbf{4 0 , 5 2}$ & $\mathbf{9 , 4 0 9}$ \\
\cline { 2 - 4 } & Est. subvencionados & 5,03 & 2,924 \\
& Est. municipalizados & 5,35 & 2,926 \\
& Total & 4,12 & 2,858
\end{tabular}

Fuente: elaboración propia 
No se observaron diferencias significativas entre los tres tipos de establecimientos A para las estrategias didácticas, $F(2 ; 144)=2,100, p=0,05$ y tampoco se observaron diferencias en cuanto al ambiente general de la sala de clases y la evaluación de los aspectos curriculares y de desarrollo de la alfabetización, $F(2 ; 144)=0,519, p=0,05$, pero se observa una calidad de ambiente letrado significativamente mayor en las escuelas privadas.

\section{Discusión}

De los datos expuestos se constata, por una parte, la baja cantidad, calidad y variedad de material de alfabetización existente en las salas observadas y, por otra, la falta de uso de estrategias de calidad orientadas a promover la alfabetización inicial. En las salas observadas se puede decir que existían menos de quince libros de buena calidad, variados en temática y dificultad, lo que redunda en menos instancias para que los niños conozcan un lenguaje más sofisticado o experimenten situaciones de interacción verbal más complejas. A lo anterior se suma que en 95 de las 147 salas no se apreció uso de libros, es decir, no se observaron educadoras leyendo a los niños ni a los niños leyendo o mirando libros en grupos pequeños o en forma individual. La ausencia de instancias y entornos de lectura constituye un factor de riesgo respecto a posibles dificultades lectoras posteriores, ya que la exposición a un ambiente rico en lectura y lenguaje facilita su aprendizaje (Kiuru et al., 2013).

Con referencia al uso de material de escritura y al desarrollo de estrategias para promover su aprendizaje, también se evidencia baja calidad, de acuerdo con los criterios establecidos en ELLCO. Los colegios y jardines infantiles más vulnerables exhiben la menor cantidad de material y casi nula evidencia de escritura infantil, lo que redunda en una desventaja frente a los niños matriculados en centros privados. La ausencia de este tipo de experiencias puede retrasar su alfabetización en una etapa fundamental para su desarrollo que, como se ha demostrado en estudios previos, constituye una instancia para equilibrar el plano entre alumnos más y menos vulnerables al comenzar la educación formal (Callaghan \& Madelaine, 2013).

Pese a lo anterior, llama la atención la ausencia de diferencias significativas en cuanto a estrategias didácticas y calidad del ambiente general entre los tipos de establecimientos, aun cuando los privados cuentan con mayor cantidad de recursos para invertir tanto en material como en capacitación docente. Es notorio, además, que en estos es posible contratar educadoras mejor preparadas que en los subvencionados y en los municipalizados, donde las remuneraciones suelen ser significativamente inferiores (Mizala \& Romagnera, 2000).
La baja correlación positiva entre las observaciones de aula y la calidad de las actividades desarrolladas por las educadoras se podría explicar por el hecho de que la observación de sala de clases evalúa el ambiente general de enseñanza en la sala junto con el currículo, el uso y desarrollo del lenguaje y la alfabetización, aspectos relacionados con las actividades que las educadoras llevan a cabo con los niños. Estos resultados están en la misma línea de lo examinado en cuanto a que un adecuado ambiente letrado no solo se consigue con buenos materiales de lectura y escritura, sino que requiere interacciones intencionadas que promuevan el uso del lenguaje, de actividades auténticas de lectura y escritura y de un currículo orientado a desarrollar aquellas habilidades que facilitarán el aprendizaje de la lectura y la escritura (Hughes \& Kwok, 2007; Jerome, Hamre \& Pianta, 2009; Chatterji, 2006; Connor, Morrison \& Katch, 2004, entre otros).

¿Qué hace, entonces, que la baja calidad de la docencia preescolar cruce todos los estratos socioeconómicos? En parte, la respuesta se podría encontrar en la falta de preparación de las educadoras, como lo ha reflejado la evaluación Inicia, aplicada desde 2011 a los egresados de las carreras de educación en Chile. Los últimos resultados muestran que apenas un $10 \%$ de los egresados domina los temas disciplinarios y pedagógicos de la carrera, incluidos aspectos como aprendizaje y desarrollo, diseño e implementación de la enseñanza o la profesión docente en el país (Mineduc, 2013). En el plano específico de la estimulación y el desarrollo del lenguaje, los conocimientos acerca del desarrollo del niño, el lenguaje y la alfabetización son componentes fundamentales a la hora de propiciar ambientes letrados favorables (Darling-Hammond, 2006). Para mejorar la calidad de la formación didáctica de las educadoras de párvulos, las instituciones formadoras han de centrar sus esfuerzos por hacer visibles a las futuras docentes aquellas estrategias didácticas necesarias para potenciar la alfabetización inicial. El modelaje de estrategias y el uso de material de lectura y escritura en contextos reales durante la formación universitaria favorecerán la creación de un ambiente letrado rico en estímulos y la existencia de instancias para adquirir progresivamente las habilidades que favorecerán el aprendizaje de la lectura y la escritura. Por ejemplo, aprovechar la lectura de cuentos para desarrollar la comprensión auditiva mediante preguntas bien formuladas y exponer a los niños a estructuras gramaticales y a un léxico variado promueve el desarrollo de la capacidad de hacer inferencias y de la comprensión lectora en general (Lepola, Lynch, Laakkonen, Silvén \& Niemi, 2012). Por otra parte, respecto a mejorar significativamente la calidad del ambiente letrado en los establecimientos, parece propicio que, al considerar la prioridad que se está otorgando al 
tema de la cobertura preescolar en Chile, no se ignoren las características físicas y materiales de las aulas. Promover el lenguaje, la lectura y la escritura por medio de un repertorio extenso y variado de libros atractivos para los niños, bien expuestos para que sean de fácil acceso parece algo evidente; sin embargo, muchas veces olvidado. Tener espacios donde se lea y se escriba que sean similares a los de la vida real ayuda a que los niños, desde muy pequeños, comprendan y valoren la alfabetización como algo inherente a la escuela y al mundo que les rodea. Estos ambientes deben estar preparados de manera intencional para que educadores y niños puedan entablar conversaciones, leer y escuchar cuentos de manera comprensiva e iniciarse en el aprendizaje de la lectura y la escritura. Un currículo orientado a aprovechar cada minuto permitirá experiencias de lenguaje enriquecedoras, en especial para quienes provienen de hogares donde hay menos intervenciones verbales o menos experiencias de alfabetización. Todo ello implica un trabajo mancomunado entre diseñadores de currículo preescolar, instituciones de educación superior encargadas de formar a los futuros educadores y políticas públicas que fomenten la educación temprana de calidad.

\section{Conclusiones}

El presente estudio permitió validar ELLCO, un instrumento para la observación del lenguaje y de la alfabetización en educación preescolar, con el fin de evaluar la calidad del ambiente letrado y de las estrategias didácticas utilizadas en dicho ciclo de la educación en Chile. El estudio constató que la calidad del ambiente de las salas de clase y de las estrategias didácticas es baja, al menos según los parámetros establecidos en ELLCO. La mala calidad del ambiente letrado se evidenció con la falta de materiales de lectura y escritura apropiados para la edad y los intereses de los niños, la ausencia de material impreso que constatara el trabajo en alfabetización emergente por parte de los alumnos y la carencia de lugares específicos en la sala para este tipo de tareas. La observación y el registro de las actividades desarrolladas por las educadoras mostraron que no cuentan con estrategias que promuevan el desarrollo de la alfabetización de manera consistente. Ambos aspectos constituyen desafíos pendientes tanto en la educación preescolar como en la formación inicial docente y que, en el caso chileno, requieren atención focalizada en el corto plazo. Como se mencionó, en los últimos años los esfuerzos se han puesto en ampliar la cobertura de la educación preescolar chilena; sin embargo, es urgente asignar recursos humanos y financieros a mejorar significativamente la calidad de la educación que se imparte en dichos lugares. La alfabetización temprana desempeña un rol fundamental, pues permite compensar los déficits lingüísticos que los niños de nivel socioeconómico bajo traen a la escuela (Dickinson \& Snow, 1987; Hart \& Risley, 1995; Hemphill \& Tivnan, 2008) y, si ellos no son atendidos a tiempo, Chile continuará ubicándose en el fondo de la tabla en materia de calidad de la educación y del capital humano.

La investigación desarrollada presenta limitaciones que es necesario tener en cuenta. En primer lugar, la muestra seleccionada solo incluye establecimientos de una región urbana del país, por lo que no constituye una muestra representativa de la realidad nacional ni pueden establecerse generalizaciones a partir de los resultados obtenidos. Como estudio exploratorio, el objetivo fue contar con una imagen de lo que ocurre en algunas salas de educación preescolar en la región, a modo de primera aproximación a un tema de interés nacional en un momento en el que se anuncian medidas importantes en la cobertura de educación en parvulario. En segundo 
lugar, como todo estudio naturalista, a pesar de entrenar a los observadores y obtener buenos índices de fiabilidad en la codificación de lo examinado, siempre existe espacio para la subjetividad a la hora de evaluar lo que se estudia. Pese a ello, ELLCO favoreció el registro de datos cuantitativos y cualitativos de manera ordenada y en torno a parámetros bien definidos.

La importancia de la educación preescolar, en particular en orden al desarrollo de habilidades de alfabetización, es foco de interés en la política pública y, por ende, se requieren más estudios que contribuyan a comprender mejor de qué manera los ambientes de los establecimientos y las estrategias didácticas empleadas por las educadoras facilitan la adquisición de disposiciones, habilidades y hábitos que ayuden en el desarrollo de la lectura y la escritura. En esta línea, es necesario investigar aspectos como los tipos de interacciones discursivas que ocurren en el aula preescolar, la intencionalidad de actividades de lectura y escritura y el modo en que se desarrollan. Conocer con mayor profundidad la manera en que las educadoras trabajan la conciencia fonológica y el principio alfabético puede contribuir a determinar dónde están los nudos críticos que inciden en que un porcentaje importante de niños presente dificultades de decodificación y comprensión lectora a lo largo de su vida escolar. Investigaciones acerca de cómo las estrategias didácticas y el entorno letrado favorecen su adquisición pueden ayudar a asignar recursos humanos y materiales de manera más eficiente.

Es evidente que los hallazgos del presente estudio son consistentes con los encontrados en investigaciones anteriores (Centro de Estudios de Desarrollo y Estimulación Psicosocial, 1997; Eyzaguirre \& Fontaine, 2008; Villalón et al., 2002; Villalón et al., 2005). Lo preocupante es que el problema cruza los distintos niveles socioeconómicos, por lo que contar con más recursos no garantiza que la calidad de la enseñanza, al menos en lo que respecta a la alfabetización, sea mayor. Con ello también se confirma el impacto positivo que tiene un buen profesor en la calidad de los aprendizajes de sus alumnos y, por ende, la tarea pendiente es mejorar la formación docente lo antes posible.

\section{Sobre los autores}

Pelusa Orellana-García es profesora asociada y decana de la Facultad de Educación, Universidad de los Andes. Investigadora en el área de diagnóstico de dificultades de lectura, teorías de la lectura y estrategias de enseñanza.

Carolina Melo-Hurtado es profesora asistente y directora de posgrados de la Facultad de Educación, Universidad de los Andes. Investigadora en el área de educación inicial, lectura y diagnóstico de dificultades. Doctoranda en Educación, Universidad de Virginia.

\section{Referencias}

Bravo, L., Villalón, M., \& Orellana, E. (2004). Los procesos cognitivos y el aprendizaje de la lectura inicial: diferencias cognitivas entre buenos lectores y lectores deficientes. Estudios Pedagógicos, (30), 7-19.

Brislin, R. W. (1970). Back-Translation for Cross-Cultural Research. Journal of Cross- Cultural Psychology, 1 (3), 185-216.

Callaghan, G., \& Madelaine, A. (2013). Leveling the Playing Field for Kindergarten Entry: Research Implications for Preschool Early Literacy Instruction. Australasian Journal of Early Childhood, 37 (1), 13-23.

Castejón, L., González-Pumariega, S., \& Cuetos, F. (2011). Adquisición de la fluidez en la lectura de palabras en una muestra de niños españoles: un estudio longitudinal. Infancia y Aprendizaje, 34 (1), 19-30.

Centro de Estudios de Desarrollo y Estimulación Psicosocial [CEDEP]. (1997). Evaluación del impacto de la educación parvularia y la educación en el primer ciclo básico sobre rendimiento. Estudio longitudinal, 1994-1996. Santiago: Autor.

Chatterji, M. (2006). Reading Achievement Gaps, Correlates and Moderators of Early Reading Achievement: Evidence from the Early Childhood Longitudinal Study (ECLS) Kindergarten to First Grade Sample. Journal of Educational Psychology, 98 (3), 489-507.

Connor, C. M., Morrison, F. J., \& Slominski, L. (2006). Preschool Instruction and Children's Literacy Skill Growth. Journal of Educational Psychology, 98 (4), 665-689.

Connor, C. M., Morrison, F., \& Katch, L. (2004). Beyond the Reading Wars: Exploring the Effect of Child-Instruction Interactions on Growth in Early Reading. Scientific Studies of Reading, 8 (4), 305-336

Cunningham, A. E., Zibulsky, J., \& Callahan, M. D. (2009). Starting Small: Building Preschool Teacher Knowledge that Supports Early Literacy Development. Reading \& Writing, 22, 487-510.

Darling-Hammond, L. (2006). Teacher Education: The Usefulness of Multiple Measures for Assessing Programs. Journal of Teacher Education, 57 (2), 120-138.

Dickinson, D. K., \& Snow, C. E. (1987). Interrelationships among Prereading and Oral Language Skills in Kindergarten from Two Social Classes. Early Childhood Research Quarterly, 2, 1-25.

Domitrovich, C. E., Gest, S. D., Gill, S., Bierman, K. L., Welsh, J. A., \& Jones, D. J. (2009). Fostering High Quality Teaching With an Enriched Curriculum 
and Professional Development: Head Start REDI. American Educational Research Journal, 46 (2), 567-597.

Eyzaguirre, B., \& Fontaine, L. (2008). Las escuelas que tenemos. Santiago: Centro de Estudios Públicos.

Eyzaguirre, B., \& Le Foulon, C. (2002). La calidad de la educación chilena en cifras. Estudios Públicos 84, 85-204.

García-Huidobro, J. E. (2006). Formación inicial de educadoras(es) de párvulos en Chile. En Expansiva (editor de la serie), en foco: 80, 1-26. Recuperado de www.expansiva.cl

Gjems, L. (2013). Teaching in ECE: Promoting Children's Language Learning and Cooperation on Knowledge Construction in Everyday Conversations in Kindergarten. Teaching and Teacher Education, 29, 39-45.

Greenfield, Spira, E., Storch Bracken, S., \& Fischel, J. E. (2005). Predicting Improvement after First-Grade Reading Difficulties: The Effects of Oral Language, Emergent Literacy, and Behavior Skills. Developmental Psychology, 41 (1), 225-234.

Guo, Y., \& Justice, L. (2010). The Literacy Environment of Preschool Classrooms: Contributions to Children's Emergent Literacy Growth. Journal of Research in Reading, 35 (3), 308-327.

Hart, B., \& Risley, T. R. (1995). Meaningful Differences in the Everyday Experience of Young American Children. Baltimore: Brookes.

Heckman, J. J. (2004). Invest in the Very Young. Recuperado de http://www.child-encyclopedia.com/ documents/HeckmanANGxp.pdf

Hemphill, L., \& Tivnan, T. (2008). The Importance of Early Vocabulary for Literacy Achievement in High-Poverty Schools. Journal of Education for Students Placed at Risk, 13, 426-451.

Hughes, J., \& Kwok, O.-M. (2007). Influence of Student-Teacher and Parent-Teacher Relationships on Lower Achieving Readers' Engagement and Achievement in the Primary Grades. Journal of Educational Psychology, 99 (1), 39-51.

Jerome, E. M., Hamre, B. K., \& Pianta, R. C. (2009). Teacher-Child Relationships from Kindergarten to Sixth Grade: Early Childhood Predictors of Teacher-Perceived Conflict and Closeness. Social Development, 18 (4), 915-945.

Justice, L. M., Meier, J., \& Walpole, S. (2005) Learning New Words from Storybooks. An Efficacy Study with At-Risk Kindergartners. Language, Speech, and Hearing Services in Schools, 36, 17-32.

Justice, L., \& Kaderavek, J. (2004). Embedded-Explicit Emergent Literacy Intervention I: Background and Description of Approach. Language, Speech, and Hearing Services in Schools, 35, 201-211.
Justice, L., Kaderavek, J., Fan, X, Sofka, A., \& Hunt, A. (2009). Accelerating Preschoolers' Early Literacy Development through Classroom-Based Teacher-Child Storybook Reading and Explicit Print Referencing. Language, Speech, and Hearing Services in Schools, 40, 67-85.

Kiuru, N., Lerkkanen, M-K., Niemi, P., Poskiparta, E., Ahonen, T., Poikkeus, A-M., Nurmi, J-E. (2013). The Role of Reading Disability Risk and Environmental Protective Factors in Children's Reading Skills in Grade 4. Reading Research Quarterly, 48 (4), 349-368

Lepola, J., Lynch, J., Laakkonen, E., Silvén, M., \& Niemi, P. (2012). The Role of Inference Making and Other Language Skills in the Development of Narrative Listening Comprehension in 4-6-YearOld Children. Reading Research Quarterly, 47 (3) 259-282.

Lira, M. I., \& Rodríguez, S. (1997). El lenguaje en preescolar de nivel socioeconómico bajo. Exploración de sus características. Santiago: Fondecyt.

LoCasale-Crouch, J., Konold, T., Pianta, R., Howes, C., Burchinal, M., Bryant, D., Clifford, D., Early, D., \& Barbarin, O. (2007). Observed Classroom Quality Profiles in State-Funded Pre-Kindergarten Programs and Associations with Teacher, Program, and Classroom Characteristics. Early Childhood Research Quarterly, 22 (1), 3-17.

Mather, N., Bos, C., \& Babur, N. (2001). Perceptions and Knowledge of Preservice and Inservice Teachers about Early Literacy Instruction. Journal of Learning Disabilities, 34 (5), 472-482.

McGinty, A. S. Justice, L. M., Piasta, S. B., Kaderavek, J., \& Fan, X. (2012). Does Context Matter? Explicit Print Instruction During Reading Varies in Its Influence by Child and Classroom Factors. Early Childhood Research Quarterly, 27 (1), 77-89.

Melhuish, E. C., Sylva, K., Sammons, P., Siraj-Blatchford, I., Taggart, B., \& Phan, M. (2008). Effects of the Home Learning Environment and Preschool Center Experience upon Literacy and Numeracy Development in Early Primary School. Journal of Social Issues, 64 (1), 95-114.

Ministerio de Educación de Chile. (2011). Resultados PISA 2009 Chile. Competencias de los Estudiantes Chilenos de 15 años en Lectura, Matemáticas y Ciencias. Santiago: Autor.

Ministerio de Educación de Chile. (2013). Evaluación diagnóstica Inicia. Resultados Institucionales 2012. CPEIP + INICIA. Santiago: Autor.

Mizala, A., \& Romagnera, P. (2000). Remuneraciones y los profesores en Chile. Santiago: Centro de Economía Aplicada, Universidad de Chile.

Mol, S. E., \& Bus, A. G. (2011). To Read or Not to Read: A Meta-Analysis of Print Exposure from Infancy 
to Early Adulthood. Psychological Bulletin, 137 (2), 267-296

Morrow, L. M. (1982). Relationships between Literacy Programs, Library Corner Design, and Children's Use of Literature. Journal of Educational Research, 75, 339-344.

Neuman, S. B., \& Cunningham, L. (2009). The Impact of Professional Development and Coaching on Early Language and Literacy Instructional Practices. American Educational Research Journal, 46 (2), 532-566.

Pianta, R. C., LaParo, K. M., \& Hamre, B. K. (2008). Classroom Assessment Scoring System. Baltimore: Paul H. Brookes.

Pressley, M., Rankin, J., \& Yokoi, L. (1996). A Survey of Instructional Practices of Primary Teachers Nominated as Effective in Promoting Literacy. The Elementary School Journal, 96 (4), 363-384.

Roskos, K. A., \& Neuman, S. B. (2001). Environment and Its Influences for Early Literacy Teaching and Learning. En S. B. Neuman \& D. K. Dickinson (eds.), Handbook of Early Literacy Research. Mahwah: Erlbaum.

Rupley, W., Blair, T., Nichols, W. D. (2009). Effective Reading Instruction for Struggling Readers. The Role of Direct/Explicit Instruction. Reading \& Writing Quarterly, 25 (2-3), 125-138.

Senechal, M., Ouellette, G., \& Rodney, D. (2006). The Misunderstood Giant: On the Predictive Role of Early Vocabulary to Future Reading. En S. B. Neuman \& D. Dickinson (eds.), Handbook of Early Literacy Research, Vol. 2. Nueva York: Guilford Press.

Skibbe, L. E., Connor, C. M., Morrison, F. J., \& Jewkes, A. M. (2011). Schooling Effects on Preschoolers' Self-Regulation, Early Literacy, and Language Growth. Early Childhood Research Quarterly, 26 (1), 42-49.

Smith, M. W., \& Dickinson, D. K. (2002). User's Guide to the Early Language and Literacy Classroom Observation toolkit. Baltimore: Brookes.

Smith, M. W., Brady, J. P., \& Clark-Chiarelli, N. (2002). ELLCO K-3 Research Edition. Baltimore: Paul H. Brookes Publishing.

Snow, C., Burns, M., \& Griffin, P. (1998). Preventing Reading Difficulties in Young Children. Washington: National Academy Press.
Storch, S. A., \& Whitehurst, G. J. (2002). Oral Language and Code-Related Precursors to Reading: Evidence from a Longitudinal Structural Model. Developmental Psychology, 38 (6), 934-947.

Strasser, K., \& Lissi, M. R. (2009). Home and Instruction Effects on Emergent Literacy in a Sample of Chilean Kindergarten Children. Scientific Studies of Reading, 13 (2), 175-204.

Strasser, K., Lissi, M. R., \& Silva, M. (2009). Gestión del tiempo en 12 salas chilenas de kindergarten: recreo, colación y algo de instrucción. Psykhe, 18 (1), 85-96.

Treviño, E., Toledo, G., \& Gempp, R. (2013). Calidad de la educación parvularia: las prácticas de clase y el camino a la mejora. Pensamiento Educativo, 50 (1), 40-62.

Villalón, M., Rojas, C., Föster, C., Valencia, E., Cox, P., $\&$ Volante, P. (2011). Resultados de la enseñanza de estrategias de lectura y escritura en la alfabetización temprana de niños con riesgo social. Estudios sobre Educación, 21, 159-179.

Villalón, M., Silva, M., Razmilic, T., \& Swartz, S. L. (2005). AlLEM Programme: A Long-Term Intervention to Promote Literacy Learning in LowPerforming Primary Schools in Chile. Early Years, 25 (2), 97-111.

Villalón, M., Suzuki, E., Herrera, M. O., \& Mathiesen, M. E. (2002). Quality of Chilean Early Childhood Education from an International Perspective. International Journal of Early Years Education, 10 (1), 49-59.

Wasik, B., \& Bond, M. A. (2001). Beyond the Pages of a book: Interactive Book Reading and Language Development in Preschool Classrooms. Journal of Educational Psychology, 93 (2), 243-250.

Whitehurst, G. J., \& Lonigan, C. J. (1998). Child Development and Emergent Literacy. Child Development, 69 (3), 848-872.

Zill, N., \& Resnick, G. (2006). Emergent Literacy of Low-Income Children in Head Start: Relationships with Child and Family Characteristics, Program Factors and Classroom Quality. En D. K. Dickinson \& S. B. Neuman (eds.), Handbook of Early Literacy Research. Nueva York: The Guilford Press. 